\title{
ASYMPTOTIC ANALYSIS OF A CRACK IN A POWER- LAW MATERIAL UNDER COMBINED IN-PLANE AND OUT-OF-PLANE SHEAR LOADING CONDITIONS
}

\author{
J. PAN \\ Mechanical Engineering and Applied Mechanics. The University of Michigan, \\ Ann Arbor, MI 48109, U.S.A.
}

(Received 19 October 1988 ; in revised form 20 April 1989)

\begin{abstract}
ASYMPTOTIC crack-tip fields for power-law hardening materials under combined in-plane and out-of-plane shear loading conditions are investigated. The governing equations of the asymptotic crack-tip fields are formulated from two stress functions, one for the in-plane stresses and the other for the out-of-plane shear stresses. These stress functions are of separable functional forms of $r$ and $\theta$, which represent the polar coordinates centered at the crack tip. Perturbations of the governing equations were carried out. The singular behavior and the angular functions of the crack-tip in-plane and the out-of-plane shear stresses obtained from the perturbation analysis agree well with the results of the finite element computations of PAN and SHIH (J. Mech. Phys. Solids 38, 161, 1990;J. appl. Mech, in press, 1989). In particular, the results from the perturbation analysis indicate that the singularity of the mode I in-plane stresses is slightly stronger than that of the mode III out-of-plane shear stresses under combined mode I and III conditions, whereas the singularity of the mode III out-of-plane shear stresses is slightly stronger than that of the mode II in-plane stresses under combined mode II and III conditions.
\end{abstract}

\section{INTRODUCTION}

AsYMPTOTIC plane-strain and plane-stress crack-tip fields for power-law materials and perfectly plastic materials have been obtained under pure mode I and pure mode II conditions (HUTCHINSON, 1968a,b; RICE and ROSENGREN, 1968; RICE, 1968a) and under combined mode I and II conditions (SHIH, 1973, 1974 ; DONG and PAN, 1989a,b). RICE $(1967,1968$ b) presented asymptotic crack-tip fields for power-law materials and perfectly plastic materials under mode III conditions. The asymptotic plane-strain and plane-stress crack-tip fields for power-law hardening and perfectly plastic anisotropic materials were given in HAYASHI (1979), PAN and SHIH $(1986,1988)$ and PAN (1986, 1988). RICE and NIKOLIC (1985) and RICE (1987) presented the asymptotic crack-tip fields for elastic-perfectly plastic single crystals. The asymptotic plane-strain and plane-stress crack-tip fields for pressure-sensitive dilatant materials were given in $\mathbf{L I}$ and PAN (1989a,b). These solutions of the crack-tip fields for power-law hardening materials are expressed in separable forms of $r$ and $\theta$, which are the polar coordinates centered at the crack tip. An excellent review of the singular crack-tip fields in nonlinear 
materials and their relevance to the $J$-based (RICE, 1968a) phenomenological nonlinear fracture mechanics can be found in HUTCHINSON (1983).

For a solid containing a three-dimensional crack under multiaxial loading conditions, the crack front generally is subjected to combined mode I, II and III loading conditions. Finite element solutions of the crack-tip fields for power-law hardening deformation plasticity materials under combined in-plane and out-of-plane shear loading and small-scale yielding conditions were presented in PAN and SHIH $(1989,1990)$. They showed that for the length scales of practical interest within the plastic zone, the singular behavior of the in-plane stresses differs from that of the out-of-plane shear stresses, and these stresses generally cannot be expressed in separable functional forms of $r$ and $\theta$.

To understand the asymptotic singular crack-tip fields and to substantiate further the results of the finite element computations of PAN and SHIH $(1989,1990)$, in this paper we formulate the governing equations for the asymptotic crack-tip fields in power-law hardening materials with the assumption of different singularities in $r$ for the in-plane and out-of-plane shear stresses. The governing equations are then perturbed, and numerical solutions of the perturbed equations are obtained. These numerical solutions are shown to be in good agreement with the results of the finite element computations of PAN and SHIH $(1989,1990)$.

\section{Hutchinson-Rice-Rosengren (HRR) CRaCK-tip FieldS}

To describe the elastic-plastic behavior of the materials, we adopt the RambergOsgood law, which is widely employed for fitting uniaxial tensile stress-strain relations :

$$
\frac{\varepsilon}{\varepsilon_{0}}=\frac{\sigma}{\sigma_{0}}+\alpha\left(\frac{\sigma}{\sigma_{0}}\right)^{n}
$$

where $\varepsilon$ is the tensile strain, $\sigma$ is the tensile stress, $\varepsilon_{0}$ and $\sigma_{0}$ are the reference strain and reference stress (usually we take the yield stress for $\sigma_{0}$ and $\varepsilon_{0}=\sigma_{0} / E$, where $E$ is Young's modulus), $\alpha$ is a material constant and $n$ is the hardening exponent. Within the context of the small-strain approach, (2.1) can be generalized using linear elasticity theory and $J_{2}$ deformation plasticity theory. The strains $\varepsilon_{i j}$ are now written as the sum of an elastic part $\varepsilon_{i j}^{p}$ and a volume-preserving plastic part $\varepsilon_{i j}^{p}$ :

$$
\varepsilon_{i j}=\varepsilon_{i j}^{e}+\varepsilon_{i j}^{p},
$$

where

$$
\varepsilon_{i j}^{e}=\frac{(1+v)}{E} s_{i j}+\frac{(1-2 v)}{3 E} \sigma_{k k} \delta_{i j}
$$




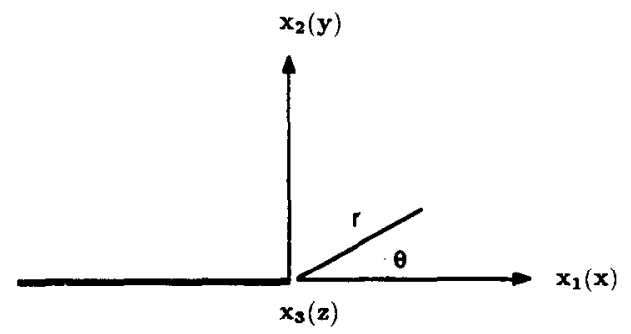

Fig. 1. Conventions at a crack tip.

$$
\varepsilon_{i j}^{p}=\frac{3}{2} \alpha\left(\frac{\sigma_{e}}{\sigma_{0}}\right)^{n-1} \frac{s_{i j}}{\sigma_{0}}
$$

where $v$ is Poisson's ratio, $s_{i j}\left(=\sigma_{i j}-\frac{1}{3} \sigma_{k k} \delta_{i j}\right)$ is the deviatoric stress and $\sigma_{e}$ $\left(=\left(3 s_{i j} s_{i j} / 2\right)^{1 / 2}\right)$ is the effective stress.

We consider a crack in a Ramberg-Osgood solid, as shown in Fig. 1, where $r$ and $\theta$ are the polar coordinates centered at the crack tip and $z$ is the coordinate perpendicular to the $(r, \theta)$ plane. Under certain remote loading conditions, as $r$ approaches 0 , the linear elastic part of the strain is negligible compared to the plastic part. The asymptotic crack-tip stress, strain and displacement fields can then be expressed as (HutchInson, 1968a,b; RICE and ROSENGREN, 1968 ; SHIH, 1973, 1874)

$$
\begin{aligned}
\sigma_{i j} & =\sigma_{0}\left(\frac{J}{\alpha \sigma_{0} \varepsilon_{0} I r}\right)^{1 /(n+1)} \tilde{\sigma}_{i j}(\theta ; n, m), \\
\varepsilon_{i j} & =\alpha \varepsilon_{0}\left(\frac{J}{\alpha \sigma_{0} \varepsilon_{0} I r}\right)^{n /(n+1)} \tilde{\varepsilon}_{i j}(\theta ; n, m), \\
u_{i}-\hat{u}_{i} & =\alpha \varepsilon_{0} r\left(\frac{J}{\alpha \sigma_{0} \varepsilon_{0} I r}\right)^{n /(n+1)} \tilde{u}_{i}(\theta ; n, m),
\end{aligned}
$$

where the dimensionless constant $I$ and the dimensionless $\theta$-variation functions $\tilde{\sigma}_{i j}, \tilde{\varepsilon}_{i j}$ and $\tilde{u}_{i}$ depend upon the hardening exponent $n$; the state of plane-strain, plane-stress or antiplane deformation; and the mode parameter $m$ (mode I, mode II, mixed mode I and II, or mode III). The constants $\hat{u}_{i}$ allow for a possible rigid body motion of the crack tip itself. The $J$-integral (RICE, 1968a) in (2.5) represents the amplitude of the singular crack-tip stress and strain fields.

Recent studies of the asymptotic crack-tip fields for power-law hardening orthotropic materials (PAN and SHIH, 1986, 1988) using Hill's quadratic anisotropic yield function (HILl, 1948) show the same type of functional forms for the crack-tip stress, strain and displacement fields as those in (2.5). The difference is that all the dimensionless functions become functions of the orthotropic material parameters in addition to the parameters mentioned earlier for $(2.5)$. LI and PAN $(1989 \mathrm{a}, \mathrm{b})$ also showed the same type of functional forms for power-law hardening pressure-sensitive dilatant materials as those in (2.5). In this case, all the dimensionless functions become 
functions of the pressure sensitivity coefficient in addition to the parameters mentioned earlier for (2.5).

Under pure in-plane or pure out-of-plane shear loading conditions, the separable functional forms of $r$ and $\theta$ for the HRR-type crack-tip fields exist and are only applicable in a small region well within the plastic zone. As shown in the finite element computations of PAN and SHIH $(1989,1990)$, under combined in-plane and out-ofplane shear loading conditions, the crack-tip stresses show a slow transition from combined modes to a more dominant mode (either an in-plane mode or an out-ofplane shear mode) for a range of length scales of practical interest within the plastic zone, depending upon the remote mode mixity of the in-plane and out-of-plane shear loading (PAN and SHIH, 1989, 1990). Therefore, it is important to remember that the relative magnitude of the in-plane stresses to the out-of-plane shear stresses within the plastic zone depends weakly upon the radial distance to the tip, in addition to the mode mixity of the remote loading. In addition, as shown in PAN and SHIH (1989, 1990), within the plastic zone the in-plane stresses and the out-of-plane shear stresses can be approximated by power-law relations with respect to $r$ where the relative magnitude of the in-plane stresses to the out-of-plane shear stresses is either small or large. This occurs in the ranges of small $r$ within the plastic zone where one of the inplane or out-of-plane shear mode is dominant due to material plasticity and/or due to small applied in-plane or out-of-plane shear loading.

\section{Assumptions and Governing Equations}

The analytical framework of the asymptotic analysis of the dominant singular neartip fields for power-law hardening materials has been detailed in HuTCHINSON (1968a,b), RiCE and ROSENGREN (1968) and RICE (1968b). Following the spirit of these works, we examine the asymptotic crack-tip fields within the plastic zone as the crack tip is approached. We consider small-scale yielding conditions where the applied load is small and the resulting plastic zone is small compared to the relevant length scale such as the crack length. Here, motivated by the results of PAN and SHIH $(1989,1990)$, we assume different singularities in $r$ for the crack-tip in-plane stresses and the cracktip out-of-plane shear stresses in the ranges of small $r$ within the plastic zone. We begin our analysis with the in-plane stress function $\phi$ and the out-of-plane stress function $\psi$. Note that the two in-plane equilibrium conditions can be satisfied when the in-plane stresses are derived from $\phi$ :

$$
\begin{aligned}
& \sigma_{r \prime}=r^{-1} \phi^{\prime}+r^{-2} \phi^{*}, \\
& \sigma_{\theta \theta}=\phi^{\prime \prime}, \\
& \sigma_{r \theta}=-\left(r^{-1} \phi^{\circ}\right)^{\prime},
\end{aligned}
$$

where ()$^{\prime}$ represents $\partial() / \partial r$ and ()$^{\circ}$ represents $\partial() / \partial \theta$. Similarly, the out-of-plane equilibrium equation is satisfied when the out-of-plane shear stresses are derived from $\psi:$ 


$$
\begin{aligned}
& \sigma_{r z}=r^{-1} \psi^{\prime}, \\
& \sigma_{\theta z}=-\psi^{\prime} .
\end{aligned}
$$

We seek solutions for $\phi$ and $\psi$ of the forms

$$
\phi=K r^{s} \widetilde{\phi}(\theta)
$$

and

$$
\psi=L r^{t} \tilde{\psi}(\theta)
$$

where $K, L, s$ and $t$ are constants, and $\tilde{\phi}(\theta)$ and $\tilde{\psi}(\theta)$ are functions of their argument $\theta$ for a given power-law material. Substituting (3.3) and (3.4) into (3.1) and (3.2), respectively, gives

$$
\begin{aligned}
& \sigma_{r r}=K r^{s-2} \tilde{\sigma}_{r r}, \\
& \sigma_{\theta \theta}=K r^{s-2} \tilde{\sigma}_{\theta \theta}, \\
& \sigma_{r \theta}=K r^{s-2} \tilde{\sigma}_{r \theta}, \\
& \sigma_{r z}=L r^{l-2} \tilde{\sigma}_{r z}, \\
& \sigma_{\theta z}=L r^{t-2} \tilde{\sigma}_{\theta z},
\end{aligned}
$$

where

$$
\begin{aligned}
& \tilde{\sigma}_{r r}=s \tilde{\phi}+\tilde{\phi}^{* *} \\
& \tilde{\sigma}_{\theta \theta}=s(s-1) \tilde{\phi} \\
& \tilde{\sigma}_{r \theta}=(1-s) \tilde{\phi} \\
& \tilde{\sigma}_{r z}=\tilde{\psi} \\
& \tilde{\sigma}_{\theta z}=(1-t) \tilde{\psi}
\end{aligned}
$$

Note that for a given power-law material, $\tilde{\sigma}_{r r}, \tilde{\sigma}_{\theta \theta}$ and $\tilde{\sigma}_{r \theta}$ are functions of $\theta$ and $s$, and $\tilde{\sigma}_{r z}$ and $\tilde{\sigma}_{\theta z}$ are functions of $\theta$ and $t$. As shown in HUTCHINSON (1968a,b), RICE and Rosengren (1968), SHIH (1973, 1974) and RiCE (1967), the value of $s$ and $t$ is

$$
s=t=\frac{2 n+1}{n+1}
$$

for either pure in-plane modes (mode I, mode II and combined mode I and II) or a pure out-of-plane shear mode (mode III). As indicated in PAN and SHIH (1989, 1990), under combined in-plane and out-of-plane shear loading conditions, the singular behavior of the in-plane stresses is different from that of the out-of-plane shear stresses in the region of practical interest within the plastic zone. Consequently, when we seek solutions of the singular crack-tip fields of the forms (3.5) and (3.6) under combined in-plane and out-of-plane shear loading conditions, the values of $s$ and $t$ are assumed to be different. Since we are seeking singular solutions in the immediate vicinity of the crack tip, we are interested in the solutions with both $s$ and $t$ being less than 2 . As $r$ approaches 0 , the in-plane mode will dominate for $s<t$ whereas the out-of-plane 
shear mode will dominate for $t<s$. Remember that the assumptions of (3.3) and (3.4) are only approximately valid when either the in-plane mode or the out-of-plane shear mode is smaller than the other.

The coupling of the in-plane plastic deformation and out-of-plane shear plastic deformation is through the effective stress $\sigma_{e}$ in the constitutive equation (2.4). The effective stress $\sigma_{e}$ in the cylindrical coordinate system is expressed as

$$
\sigma_{e}^{2}=\frac{3}{4}\left(\sigma_{r r}-\sigma_{\theta \theta}\right)^{2}+3 \sigma_{r \theta}^{2}+3 \sigma_{r z}^{2}+3 \sigma_{\theta z}^{2} .
$$

Substituting (3.5) into (3.8) gives

$$
\sigma_{e}^{2}=\left(K r^{-2}\right)^{2} \tilde{S}_{e}^{2}+\left(L r^{t-2}\right)^{2} \tilde{T}_{e}^{2},
$$

where

$$
\tilde{S}_{e}^{2}=\frac{3}{4}\left(\tilde{\sigma}_{r r}-\tilde{\sigma}_{\theta \theta}\right)^{2}+3 \tilde{\sigma}_{r \theta}^{2}
$$

and

$$
\tilde{T}_{e}^{2}=3 \tilde{\sigma}_{r z}^{2}+3 \tilde{\sigma}_{\theta *}^{2} .
$$

Note that $\widetilde{S}_{e}$ and $\widetilde{T}_{e}$ are functions of $\theta$ only. More importantly, since we assume that $s$ is not equal to $t, \sigma_{e}$ cannot be expressed as a separable function of $r$ and $\theta$, such as those stresses in (3.5). We can define the magnitude of the $\theta$-variation $\tilde{S}_{f}$ and $\tilde{T}_{c}$ to be in the order of unity. Then $K$ and $L$ represent the singularity amplitudes of the inplane stresses and the out-of-plane shear stresses, respectively.

The in-plane compatibility equation and the out-of-plane compatibility equation are

$$
\frac{\partial}{\partial r}\left(2 r \frac{\partial \varepsilon_{r \theta}}{\partial \theta}-r^{2} \frac{\partial \varepsilon_{\theta \theta}}{\partial r}\right)+r \frac{\partial \varepsilon_{r r}}{\partial r}-\frac{\partial^{2} \varepsilon_{r r}}{\partial \theta^{2}}=0
$$

and

$$
\frac{\partial}{\partial r}\left(r \varepsilon_{\theta z}\right)-\frac{\partial \varepsilon_{r z}}{\partial \theta}=0
$$

Combining (3.3)-(3.6), (3.9)-(3.13) and (2.4) gives two differential equations for $\tilde{\phi}(\theta)$ and $\tilde{\psi}(\theta)$ with a dependence on $r$. In the next two sections, we will perturb the two equations with the assumption of either $L / K \ll 1$ or $K / L \ll 1$. The lowest-order perturbed equations are two ordinary differential equations for $\tilde{\psi}(\theta)$ and $\tilde{\phi}(\theta)$. The perturbation solutions will reveal the characteristics of the final part of the transition from the mixture of the two modes to one dominant mode and the early part of the transition from one dominant mode to the mixture of two modes.

\section{Perturbation from Dominant In-pi.ane Modes}

First, we consider the cases where the contribution of the out-of-plane shear stresses to the effective stress is small compared to that of the in-plane stresses (or $L / K \ll 1$ ). Equation (3.9) can be rewritten as 


$$
\sigma_{e}=K r^{s-2} \tilde{\sigma}_{e},
$$

where $\tilde{\sigma}_{e}$ is defined as

$$
\tilde{\sigma}_{e}^{2}=\tilde{S}_{e}^{2}\left(1+\left(\frac{L}{K}\right)^{2} r^{2(1-s)} \frac{\tilde{T}_{e}^{2}}{\tilde{S}_{e}^{2}}\right) .
$$

As shown in the finite element computations of PAN and SHIH $(1989,1990)$, within the plastic zone the singularities of the in-plane stresses and the out-of-plane shear stresses differ only slightly. Therefore, $t-s$ in (4.2) can be assumed to be a small number. For small $r$ (relative to the relevant physical length scale such as the plastic zone size), $r^{2(t-s)}$ in (4.2) is finite with an order of unity. (We choose to work with the current prevailing HRR form of (2.5) with all the physical quantities instead of those dimensionless quantities used in HuTCHINson, 1968a.) Therefore, $\tilde{\sigma}_{e}$ does have a weak dependence on $r$. Since $\tilde{S}_{\mathrm{e}}$ and $\tilde{T}_{\mathrm{e}}$ are defined to have the order of unity, $(L / K)^{2}$ determines the order of magnitude of the second term on the right-hand side of (4.2). In fact, $(L / K)^{2} r^{2\langle t-s\rangle}$ can be regarded as a mixity factor of the in-plane mode and the out-of-plane shear mode. This mixity factor depends upon $r$.

Substituting (3.5) and (4.1) into (2.4) gives

$$
\begin{aligned}
& \varepsilon_{r r}=M r^{n(s-2)} \tilde{\sigma}_{e}^{n-1} \frac{1}{2}\left(\tilde{\sigma}_{r r}-\tilde{\sigma}_{\theta \theta}\right), \\
& \varepsilon_{\theta \theta}=-\varepsilon_{r r}, \\
& \varepsilon_{r \theta}=M r^{n(s-2)} \tilde{\sigma}_{e}^{n-1} \tilde{\sigma}_{r \theta}, \\
& \varepsilon_{r z}=M\left(\frac{L}{K}\right) r^{n(s-2)+(l-s)} \tilde{\sigma}_{e}^{n-1} \tilde{\sigma}_{r z}, \\
& \varepsilon_{\theta z}=M\left(\frac{L}{K}\right) r^{n(s-2)+(t-s)} \tilde{\sigma}_{e}^{n-1} \tilde{\sigma}_{\theta z},
\end{aligned}
$$

where

$$
M=\left(\frac{3}{2}\right) \propto\left(\frac{K}{\sigma_{0}}\right)^{n} .
$$

Note again that $\tilde{\sigma}_{e}$ is a nonseparable function of $r$ and $\theta$ when $t$ is not equal to $s$. Substituting (4.3) into the two compatibility equations, (3.12) and (3.13), gives two differential equations for $\tilde{\phi}(\theta)$ and $\tilde{\psi}(\theta)$ with a dependence on $r$. Assuming that the second term on the right-hand side of (4.2) is smaller than unity, we can perturb these two equations from the in-plane modes using

$$
\tilde{\sigma}_{e}^{n-1}=\tilde{S}_{e}^{n-1}\left(1+\left(\frac{L}{K}\right)^{2} \frac{n-1}{2} r^{2(t-s)} \frac{\tilde{T}_{e}^{2}}{\tilde{S}_{e}^{2}}\right) .
$$

The unperturbed equation for the in-plane modes is the same as the ordinary differential equation for $\bar{\phi}$ derived by Hurchinsun (1968a). The lowest-order perturbed equation for the out-of-plane shear mode is 


$$
(n(s-2)+1+t-s) \tilde{\sigma}_{\theta t}-\tilde{\sigma}_{r z}^{*}-\tilde{\sigma}_{r z}(n-1) \tilde{S}_{r}^{-2}\left(\frac{3}{4}\left(\tilde{\sigma}_{r r}-\tilde{\sigma}_{\theta \theta}\right)\left(\tilde{\sigma}_{r r}^{*}-\tilde{\sigma}_{\theta+\theta}^{*}\right)+3 \tilde{\sigma}_{r+\theta} \tilde{\sigma}_{r \theta}^{*}\right)=0 .
$$

Note that (4.6) is homogeneous in $\tilde{\phi}$ and $\tilde{\psi}$. In fact. we now are seeking the solutions for the out-of-plane shear mode perturbed from an in-plane mode. We first solve $\bar{\phi}$ of the unperturbed equation for an in-plane mode. The numerical procedure for solving $\delta$ and $s$ has been elaborated on in HUTCHINSON (1968a,b) and RicE and ROSENGREN (1968). Note that $s=(2 n+1) /(n+1)$ for pure in-plane modes (mode I, mode II, and mixed mode I and II). Then we can solve for $\tilde{\psi}$ based on (4.6) with the known $\widetilde{\phi}$ of a given in-plane mode.

The anti-symmetry conditions at $\theta=0$ and the stress-free boundary conditions at $\theta=\pi$ give

$$
\begin{aligned}
\tilde{\psi}(0) & =0 \\
\tilde{\psi}(\pi) & =0 .
\end{aligned}
$$

Since (4.6) is homogeneous in $\tilde{\psi}$, for any solution that satisfies the governing equation (4.6) and the boundary conditions and anti-symmetry conditions of (4.7), its multiples are also solutions. Since we are solving the equation that is homogeneous in $\tilde{\psi}$, we can arbitrarily assign $\tilde{\psi}(0)=-1$ as one of the initial conditions for numerical integration from $\theta=0$ to $\theta=\pi$.

We can select an eigenvalue $t$ and integrate the governing equation (4.6) using the fifth-order Runge-Kutta method. Then we use the Newton-Raphson method to iterate the value of $t$ to satisfy the boundary conditions at the end point, $\bar{\psi}(\pi)=0$. We select $t=(2 n+1) /(n+1)$ as the initial estimate for the eigenvalue $t$. After a few iterations, an eigenvalue $t$ can be found to satisfy the governing equation (4.6) and the boundary conditions and anti-symmetry conditions of (4.7) for each $n$. We normalize our stress solutions such that the maximum value of the $\theta$-variation $\tilde{T}_{i}$ is set at unity.

\subsection{Numerical results}

Figure 2 ( $\mathrm{a}$ and b) shows the mode III crack-tip stress solutions perturbed from mode I for $n=3$ and $n=10$, respectively. For comparison, Fig. 3 (a and b) shows the pure mode III crack-tip stress solutions for $n=3$ and $n=10$, respectively. In Fig. 2 ( $\mathrm{a}$ and $\mathrm{b}$ ), the most interesting feature of the solutions is the upturned angular functions $\tilde{\sigma}_{r z}$ and $\tilde{T}_{e}$ at large $\theta$. This feature differs drastically from the pure mode III stress solutions shown in Fig. 3 ( $a$ and b). When we compare Fig. 2(a) with Fig. 2(b), we can see that as $n$ increases, $\tilde{\sigma}_{\theta-}$ becomes almost a constant between 0 and 135 , and the ratios $\tilde{\sigma}_{t z}(\pi) / \tilde{\sigma}_{\theta z}(0)$ and $\tilde{T}_{e}(\pi) / \tilde{T}_{t}(0)$ increase.

In Table 1 , the values of $t$ are listed for various $n$ s. For comparison, we also list the values of $s$, which are the HRR values $[(2 n+1) /(n+1)]$. The numerical solution of $t$ for $n=1$ is equal to $s$, which is $\frac{3}{2}$. The angular stress functions for $n=1$ are identical to the linear elastic solutions for mode III. The agreement of our $n=1$ solution with the linear elastic solution serves as a check for our numerical procedure. The eigenvalue $t$ is slightly larger than the corresponding HRR value for each $n$ larger than 1 . The 


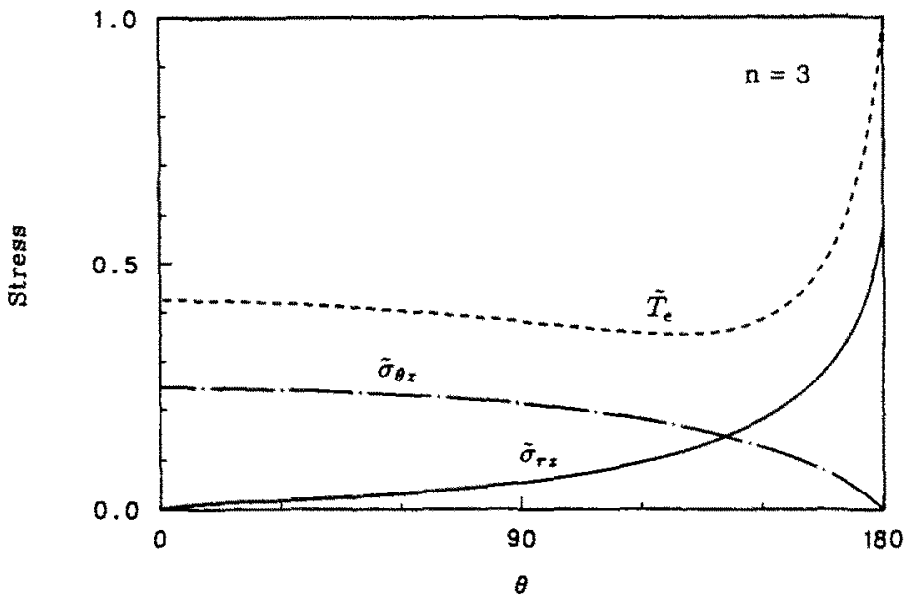

(a)

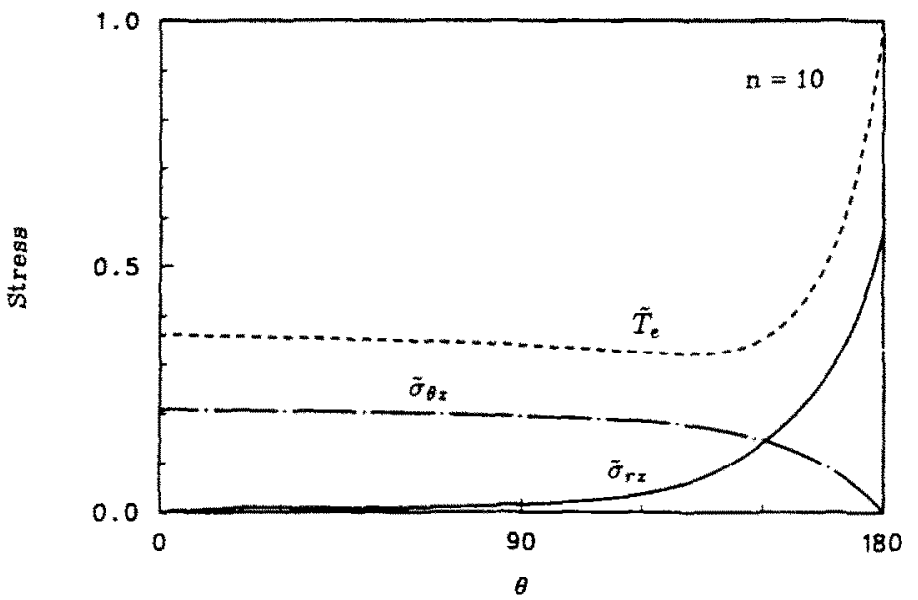

(b)

FIG. 2. The mode III crack-tip stress solutions perturbed from mode 1. (a) $n=3$, (b) $n=10$.

values of $s-2$ and $t-2$ are also listed in Table $1 ;$ these represent the degree of singularity for the in-plane stresses and the out-of-plane shear stresses. As indicated in the table, the mode III crack-tip stresses are slightly less singular than the mode I crack-tip stresses. This characteristic also was observed in the finite element computations of PAN and SHIH (1990).

PAN and SHIH $(1989,1990)$ used finite element methods to investigate the mathematical structure of the crack-tip fields within the plastic zone under small-scale yielding and remotely applied combined in-plane and out-of-plane shear $K$ fields. They used 9-node quadrilateral Lagrangian elements. Wedged-shaped elements were 


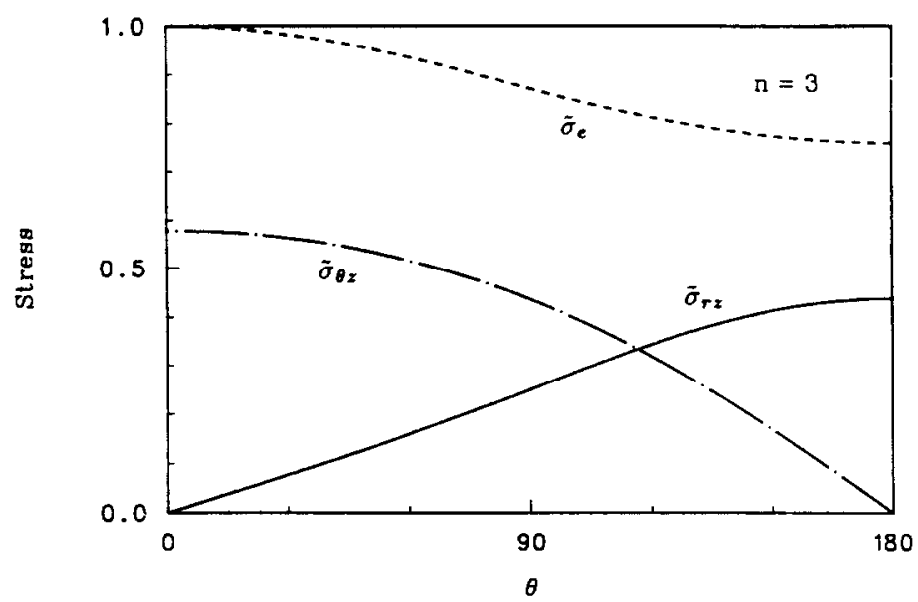

(a)

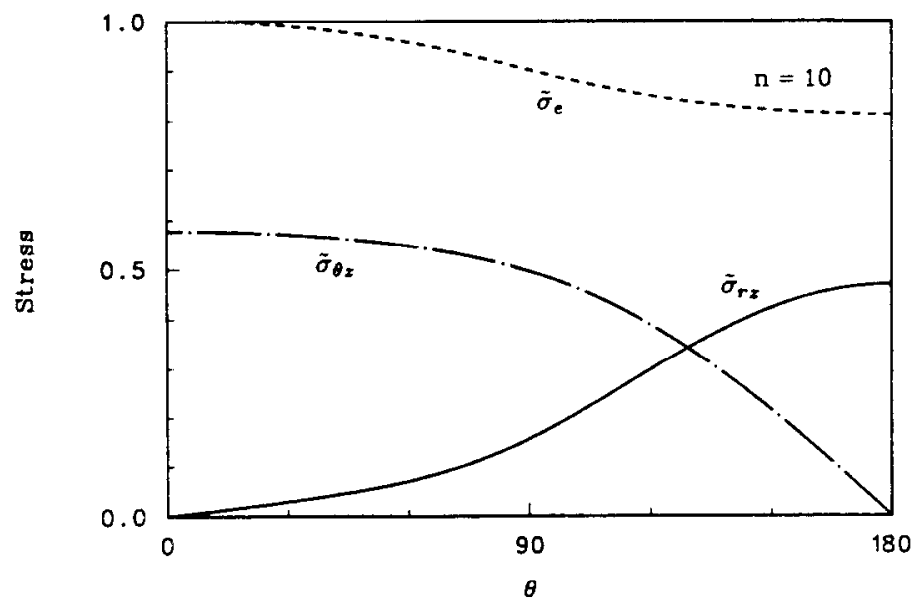

(b)

FIG. 3. The pure mode III crack-tip stress solutions. (a) $n=3$. (b) $n=10$.

TABLE 1. The values of $\mathrm{t}$ for mode III perturbed from mode $I[s=(2 \mathrm{n}+1) /(\mathrm{n}+1)]$

\begin{tabular}{rcccc}
\hline$n$ & $s$ & $s-2$ & $t$ & $t-2$ \\
\hline 1 & 1.5000 & -0.5000 & 1.5000 & -0.5000 \\
2 & 1.6667 & -0.3332 & 1.6918 & -0.3082 \\
3 & 1.7500 & -0.2500 & 1.7962 & -0.2038 \\
5 & 1.8333 & -0.1667 & 1.8878 & -0.1122 \\
10 & 1.9091 & -0.0909 & 1.9563 & -0.0437 \\
\hline
\end{tabular}




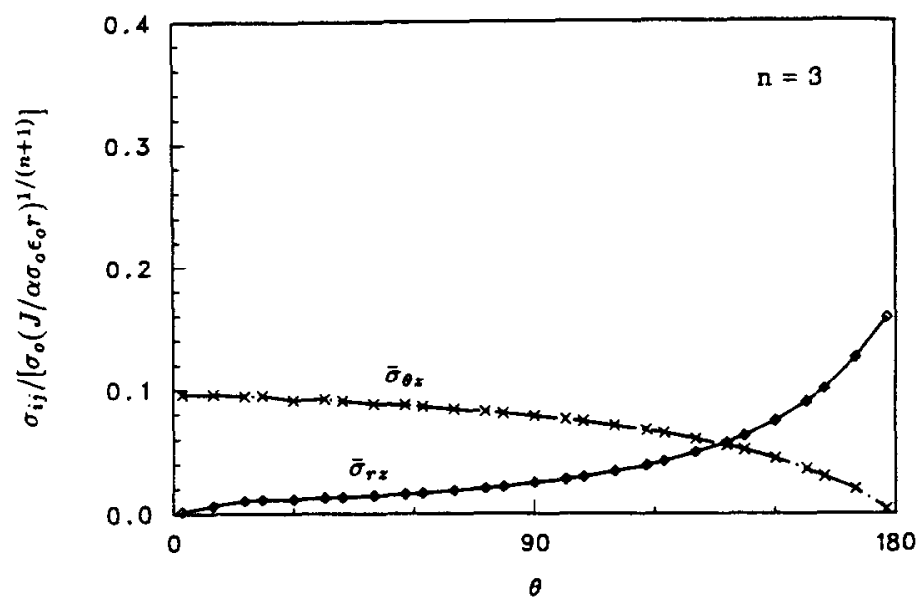

(a)

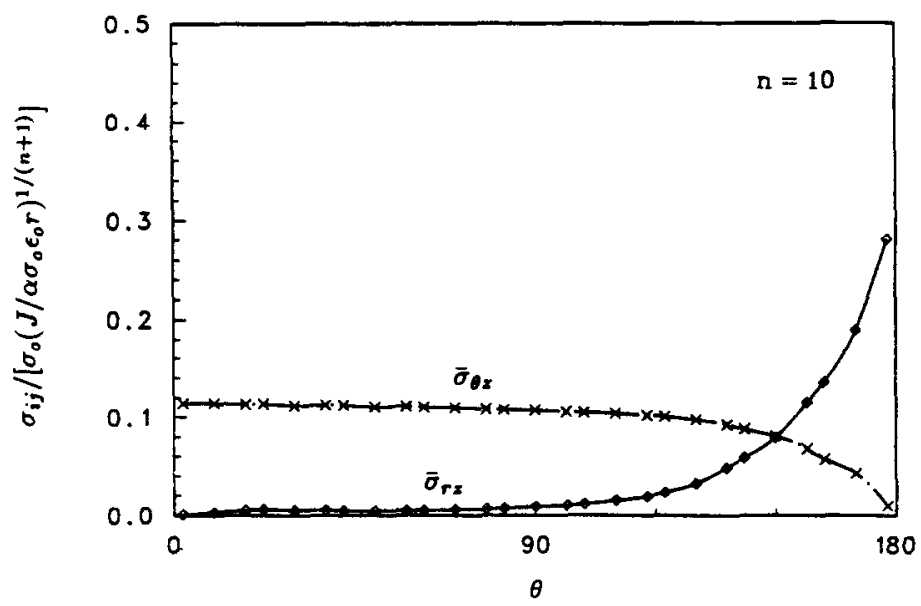

(b)

FIG. 4. The normalized mode III crack-tip stress solutions at $r / r_{p} \approx 10^{-3}$ from finite element computations under remote combined mode I and III conditions of $K_{111} / K_{1}=0.12$. (a) $n=3$, (b) $n=10$.

used in the immediate crack-tip region, which was surrounded by semi-circular strips of elements generated by a logarithmic scale. Here, we will present the crack-tip outof-plane shear stress solutions at a small radial distance (to the tip) within the plastic zone, where the contribution of the out-of-plane shear stresses to the effective stress is smaller than that of the in-plane stresses.

The normalized mode III out-of-plane shear stresses, $\bar{\sigma}_{i j}=\sigma_{i j} /\left[\sigma_{0}\left(J / \alpha \sigma_{0} \varepsilon_{0} r\right)^{1 /(n+1)}\right]$, at the Gauss points at about $r / r_{p} \approx 10^{-3}\left(r_{p}\right.$ represents the plastic zone size at $\theta=0^{\circ}$ ) are plotted in Fig. 4 (a and b) under remote loading of $K_{11} / K_{1}=0.12$. The angular 
functions of the normalized mode III out-of-plane shear stresses shown in Fig. 4 (a and b) closely resemble those of the perturbation solutions shown in Fig. 2 ( $a$ and $b$ ).

The ratio $\sigma_{\theta=} / \sigma_{\theta \theta}$ at $\theta=0$ is about 0.12 for the remote $K$ field. At a small radial distance to the tip, $r / r_{r} \approx 10^{-3}$, the ratio $\sigma_{\theta z} / \sigma_{\theta \theta}$ at $\theta=0^{\circ}$ is about 0.082 for $n=3$ and about 0.055 for $n=10$. The results indicate that due to the material plasticity, within the plastic zone the ratio of the magnitude of the out-of-plane shear stresses to the magnitude of the in-plane stresses decreases as $r$ decreases. This weaker singularity of the mode III out-of-plane shear stresses compared to that of the mode I in-plane stresses agrees with the results of our perturbation analysis. This effect is more pronounced for low-hardening materials (with large $n$ ). Furthermore, the estimated values of the singularity difference $t-s$ from these finite element computations for the cases where the contribution of the mode III out-of-plane shear stresses to the effective stress is smaller than that of the mode I in-plane stresses are in good agreement with the values listed in Table 1 .

Figure 5 ( $a$ and $b$ ) show the mode III crack-tip stress solutions perturbed from mode II for $n=3$ and $n=10$, respectively. In Fig. 5 (a and b) the perturbation solutions show a hump in $\tilde{\sigma}_{r z}$ at about $100^{\circ}$. In Fig. $5(\mathrm{~b})$ for $n=10$, this hump becomes more distinct and a second hump at about $160^{\circ}$ develops.

In Table 2, the values of $t$ and $s$ are listed for various $n$ s. As mentioned before. the values of $s$ listed in the table are the HRR values $[(2 n+1) /(n+1)]$, since we seek the solutions perturbed from mode II. As indicated in the table, for each $n$ larger than 1 , the value of $t$ is slightly smaller than the value of $s$; and the values of $t$ and $s$ are very close to each other. This indicates that the singularity of the mode III out-of-plane shear stresses is just slightly stronger than that of the mode II in-plane stresses and that the singularities of the in-plane and out-of-plane shear stresses are very close to each other under near mode II, combined mode II and III conditions. This characteristic has been also shown in PAN and SHIH (1989). In the case of $t<s$, as $r$ approaches 0 , the out-of-plane shear mode will dominate. Therefore, the perturbation solutions are only approximately valid in certain ranges of small $r$ within the plastic zone where the out-of-plane shear mode is smaller than the in-plane modes (or $(L / K)^{2}$ is small). This was indeed shown in PAN and SHIH (1989).

Figure 6 (a and b) shows the normalized mode III out-of-plane shear stresses at the Gauss points at about $r / r_{p} \approx 10^{-3}$ under remote loading of $K_{\mathrm{III}} / K_{\mathrm{II}}=0.27$ from the finite element computations of $\mathrm{P}_{\mathrm{AN}}$ and $\mathrm{SHIH}_{\mathrm{H}}$ (1989). The angular functions of the mode III out-of-plane shear stresses shown in Fig. 6 (a and b) closely resemble those of the perturbation solutions shown in Fig. 5 ( $a$ and $b$ ).

The ratio $\sigma_{\theta z} / \sigma_{r t}$ at $\theta=0$ is about 0.27 for the remote $K$ field. At a small radial distance to the tip, $r / r_{n} \approx 10^{-3}$, the ratio $\sigma_{\theta z} / \sigma_{r i}$ at $\theta=0^{\circ}$ is about 0.26 for $n=3$ and about 0.29 for $n=10$. Note that the ratio $\sigma_{\theta=} / \sigma_{r \theta}$ at $\theta=0$ increases to about 0.27 for $n=3$ and about 0.33 for $n=10$ at about $r / r_{p} \approx 10^{-11}$. The results indicate that well within the plastic zone $\left(r / r_{r}<10^{-3}\right)$ the singularity of the mode II in-plane stresses is slightly weaker than that of the mode III out-of-plane shear stresses. This characteristic agrees with the results of the perturbation analysis. We realize that our results at $r / r_{p} \approx 10^{-11}$ have no physical meaning because the current small-strain continuum approach is not applicable at this length scale. However, we are now investigating the 
Asymptotic analysis of a crack under combined loading

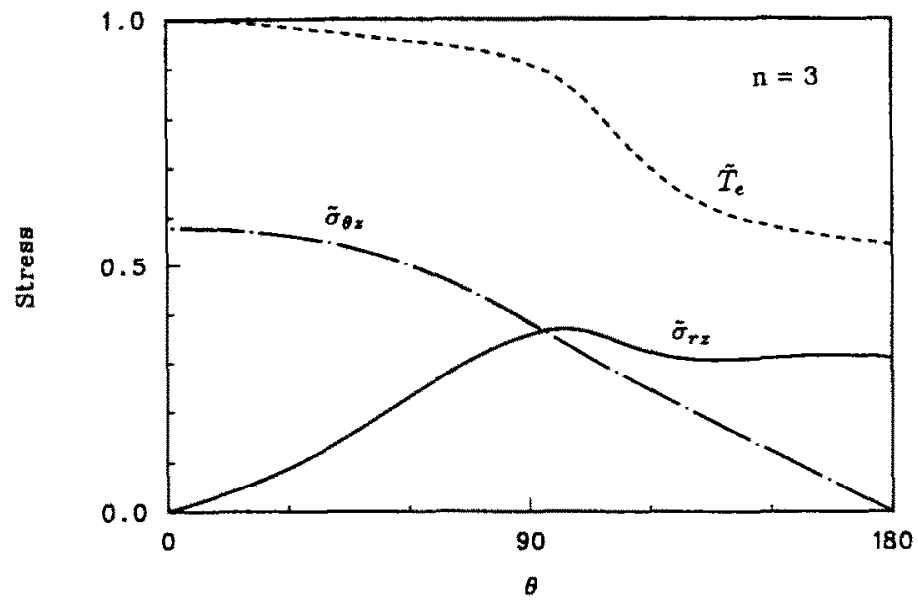

(a)

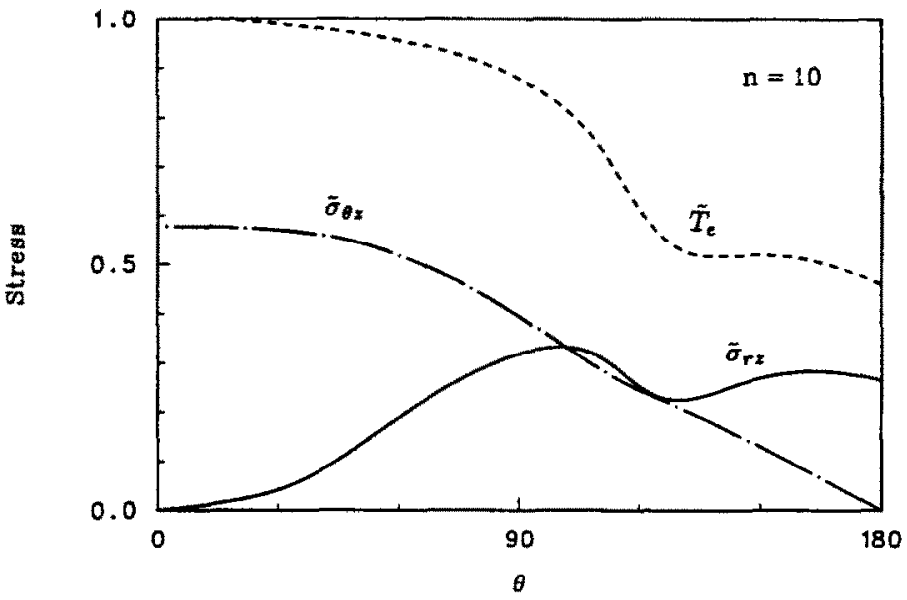

(b)

FiG. 5. The mode III crack-tip stress solutions perturbed from mode II. (a) $n=3$, (b) $n=10$.

TABLE 2. The values of $\mathrm{t}$ for mode $I I I$ perturbed from mode $I I[\mathrm{~s}=(2 \mathrm{n}+1) /(\mathrm{n}+1)]$

\begin{tabular}{rcccc}
\hline$n$ & $s$ & $s-2$ & $t$ & $t-2$ \\
\hline 1 & 1.5000 & -0.5000 & 1.5000 & -0.5000 \\
2 & 1.6667 & -0.3333 & 1.6646 & -0.3354 \\
3 & 1.7500 & -0.2500 & 1.7463 & -0.2527 \\
5 & 1.8333 & -0.1667 & 1.8282 & -0.1718 \\
10 & 1.9091 & -0.0909 & 1.9041 & -0.0959 \\
\hline
\end{tabular}




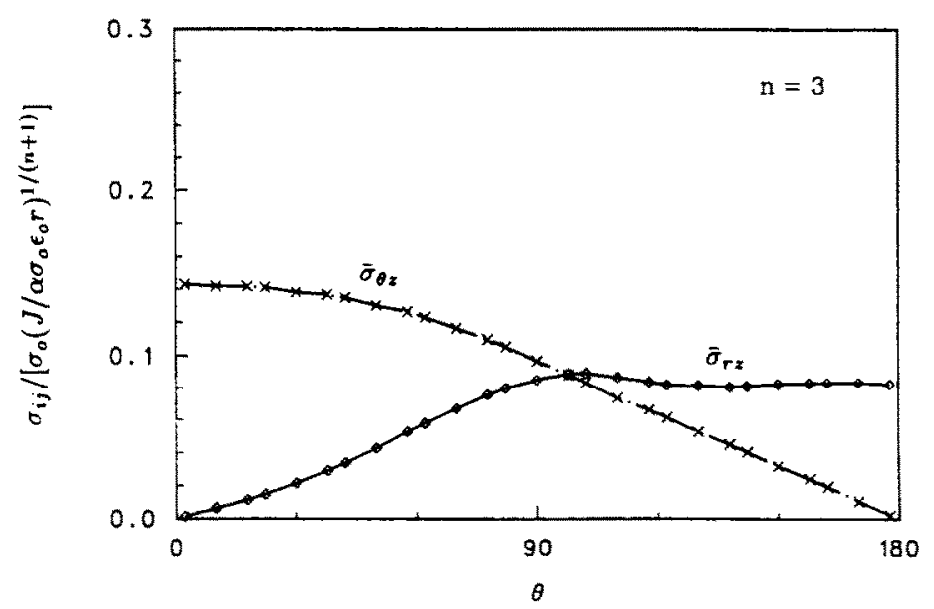

(a)

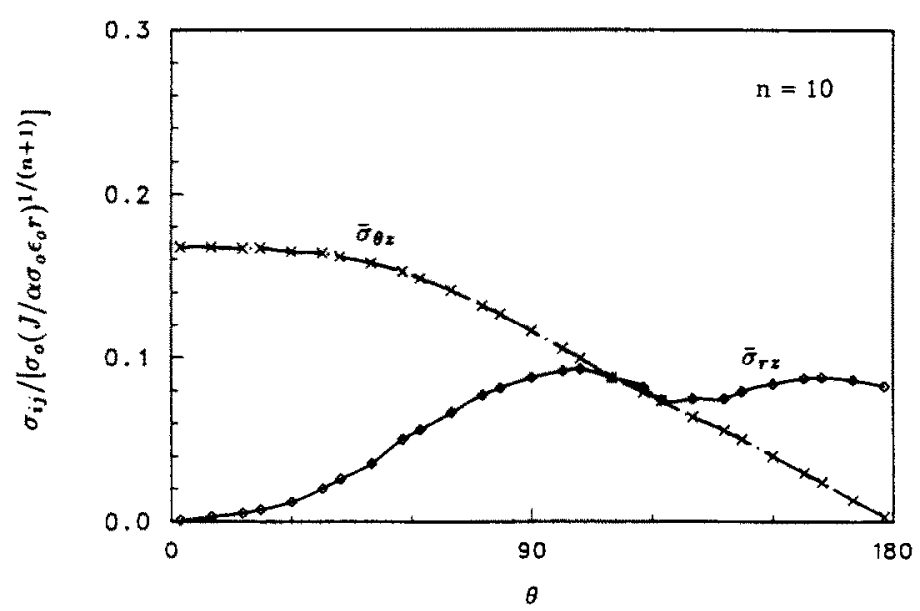

(b)

FIG. 6. The normalized mode III crack-tip stress solutions at $r / r_{n} \approx 10^{-3}$ from finite element computations under remote combined mode II and III conditions of $K_{11} / K_{11}=0.27$. (a) $n=3$, (b) $n=10$.

mathematical structure of the crack-tip fields. Therefore, we present the results to verify the results of our perturbation analysis in the mathematical sense.

The reason that the ratio $\sigma_{\theta z} / \sigma_{r \theta}$ for $n=3$ decreases a bit in the range of $r / r_{p}=1$ to $10^{-3}$ as $r$ decreases is perhaps due to the adjustment of the stresses from the elastic state to the plastic state with the decrease of $r$. Generally speaking, these finite element computations show that due to the material plasticity, well within the plastic zone the ratio of the magnitude of the out-of-plane shear stresses to the magnitude of the inplane stresses increases slightly as $r$ decreases. The effect of material plasticity is 
more pronounced for low-hardening materials (with large $n$ ). The slightly stronger singularity of the mode III out-of-plane shear stresses compared to that of the mode II in-plane stresses agrees with the results of our perturbation analysis. The estimated values of the singularity difference $t-s$ from these finite element computations for the cases where the contribution of the mode III out-of-plane shear stresses to the effective stress is smaller than that of the mode II in-plane stresses are in good agreement with the values listed in Table 2 .

\section{Perturbation from Dominant Out-of-plane Mode}

Now, we consider the cases where the contribution of the in-plane stresses to the effective stress is smaller than that of the out-of-plane shear stresses (or $K / L \ll 1$ ). Equation (3.9) can be rewritten as

$$
\sigma_{e}=L r^{t-2} \tilde{\sigma}_{e}
$$

where $\tilde{\sigma}_{e}$ is now defined as

$$
\tilde{\sigma}_{e}^{2}=\tilde{T}_{e}^{2}\left(1+\left(\frac{K}{L}\right)^{2} r^{2(s-t)} \frac{\tilde{S}_{e}^{2}}{\tilde{T}_{e}^{2}}\right)
$$

As shown in the finite element computations of PAN and SHIH $(1989,1990)$, the singularities of the crack-tip in-plane stresses and out-of-plane shear stresses differ only slightly. Therefore, $s-t$ in (5.2) can be assumed to be a small number. For small $r, r^{2(t-s)}$ in (5.2) becomes finite and has an order of unity. Therefore, $\tilde{\sigma}_{e}$ does have a weak dependence on $r$. Then, $(K / L)^{2}$ determines the order of magnitude of the second term on the right-hand side of $(5.2)$. Note that $(K / L)^{2} r^{2(s-t)}$ represents a mixity factor of the in-plane mode and the out-of-plane shear mode.

The strains can be expressed as

$$
\begin{aligned}
& \varepsilon_{r r}=N\left(\frac{K}{L}\right) r^{n(t-2)+s-1} \tilde{\sigma}_{e}^{n-1} \frac{1}{2}\left(\tilde{\sigma}_{r r}-\tilde{\sigma}_{\theta \theta}\right), \\
& \varepsilon_{\theta \theta}=-\varepsilon_{r r}, \\
& \varepsilon_{r \theta}=N\left(\frac{K}{L}\right) r^{n(t-2)+s-1} \tilde{\sigma}_{e}^{n-1} \tilde{\sigma}_{r \theta}, \\
& \varepsilon_{r z}=N r^{n(t-2)} \tilde{\sigma}_{e}^{n-1} \tilde{\sigma}_{r z}, \\
& \varepsilon_{\theta z}=N r^{n(t-2)} \tilde{\sigma}_{e}^{n-1} \tilde{\sigma}_{\theta z},
\end{aligned}
$$

where

$$
N=\left(\frac{3}{2}\right) \alpha\left(\frac{L}{\sigma_{0}}\right)^{n}
$$

Substituting (5.3) into the two compatibility equations, (3.12) and (3.13), gives two differential equations for $\tilde{\phi}(\theta)$ and $\tilde{\psi}(\theta)$ with a dependence on $r$. Assuming that the 
second term on the right-hand side of (5.2) is smaller than unity, we can perturb these two equations from the out-of-plane shear mode using

$$
\tilde{\sigma}_{e}^{n-1}=\tilde{T}_{c}^{n-1}\left(1+\left(\frac{K}{L}\right)^{2} \frac{n-1}{2} r^{2(s-t)} \frac{\tilde{S}_{e}^{2}}{\tilde{T}_{e}^{2}}\right) .
$$

The unperturbed equation for the out-of-plane shear mode is identical to the ordinary differential equation for $\bar{\psi}$ for pure mode III. Therefore, the eigenvalue $t$ should be the same as the HRR value $[(2 n+1) /(n+1)]$. The lowest-order perturbed equation for the in-plane modes is

$$
\begin{aligned}
{[2(n(t-2)+} & \left.+1+s-t)\left(\tilde{\sigma}_{r \theta}^{*}+(n-1) \tilde{\sigma}_{r \theta} \tilde{T}_{e}^{-1} \tilde{T}_{e}^{*}\right)\right]+[(n(t-2)+s-t)(n(t-2) \\
& \left.+2+s-t) \frac{1}{2}\left(\tilde{\sigma}_{r r}-\tilde{\sigma}_{\theta \theta}\right)\right]-\left[\frac{1}{2}\left(\tilde{\sigma}_{r r}^{*}-\tilde{\sigma}_{\theta \theta}^{*}\right)+(n-1)\left(\tilde{\sigma}_{r r}^{*}-\tilde{\sigma}_{\theta \theta}^{*}\right) \tilde{T}_{c}^{-1} \tilde{T}_{c}^{.}\right. \\
& \left.+(n-1)(n-2) \frac{1}{2}\left(\tilde{\sigma}_{r r}-\tilde{\sigma}_{\theta \theta}\right) \tilde{T}_{r}^{-2}\left(\tilde{T}_{c}^{*}\right)^{2}+(n-1) \frac{1}{2}\left(\tilde{\sigma}_{r r}-\tilde{\sigma}_{\theta \theta}\right) \tilde{T}_{e}^{-1} \tilde{T}_{e}^{*}\right]=0 .
\end{aligned}
$$

Note that (5.6) is homogeneous in $\tilde{\phi}$ and $\tilde{\psi}$. We now are seeking the solutions of the in-plane modes perturbed from the out-of-plane shear mode. We can first solve $\bar{\psi}$ of the unperturbed equation for the out-of-plane shear mode:

$$
(n(t-2)+1) \tilde{\sigma}_{\theta z}-\tilde{\sigma}_{r z}^{*}-\tilde{\sigma}_{r z}(n-1) \tilde{T}_{e}^{-1} \tilde{T}_{e}^{*}=0
$$

In (5.6), $\tilde{T}_{e}^{* *}$ can be obtained by differentiating (5.7). Equation (5.6) is a fourth-order ordinary differential equation for $\tilde{\phi}$ for a known solution of $\tilde{\psi}$. The numerical procedure for solving $\tilde{\phi}$ and $s$ has been elaborated on in HUTCHINSON $(1968 \mathrm{a}, \mathrm{b})$.

When we perturb from the out-of-plane shear mode, we have three different inplane modes to consider: pure mode I, pure mode II, and mixed mode I and II. We can integrate (5.6) from $\theta=0$ to $\theta=\pi$ for pure mode $I$ and pure mode II. The stressfree boundary conditions at $\theta=\pi$ are

$$
\begin{aligned}
\tilde{\phi}(\pi) & =0, \\
\tilde{\phi}^{\cdot}(\pi) & =0 .
\end{aligned}
$$

For mode I, the symmetry conditions at $\theta=0$ give

$$
\begin{aligned}
\tilde{\phi}^{*}(0) & =0, \\
\tilde{\phi}^{\cdots}(0) & =0 .
\end{aligned}
$$

For mode II, the anti-symmetry conditions at $\theta=0$ give

$$
\begin{aligned}
\tilde{\phi}(0) & =0, \\
\tilde{\phi} \cdot *(0) & =0 .
\end{aligned}
$$

For mixed mode I and II, (5.6) is solved from $\theta=-\pi$ to $\theta=\pi$. The stress-free boundary conditions are

$$
\begin{aligned}
\tilde{\phi}(-\pi) & =\tilde{\phi}(\pi)=0, \\
\tilde{\phi}^{*}(-\pi)=\tilde{\phi}^{*}(\pi) & =0 .
\end{aligned}
$$

For these modes, the symmetry or anti-symmetry conditions and/or the stress-free 
boundary conditions give two homogeneous conditions at the end points of the $\theta$ range of interest.

Since (5.6) is homogeneous in $\tilde{\phi}$, for any solution that satisfies the governing equation (5.6), as well as the boundary and/or the symmetry or anti-symmetry conditions of (5.8)-(5.11) for different modes, its multiples are also solutions. Since we are solving the equation that is homogeneous in $\tilde{\phi}$, for example, for mode $I$, we can arbitrarily assign $\tilde{\phi}(0)=1$. We can select an eigenvalue $s$ and an initial valuc of $\tilde{\phi}^{-*}(0)$. We can then integrate the governing equation (5.6) from $\theta=0$ to $\theta=\pi$ using the fourth-fifth order Runge-Kutta method with error control. Finally, we use the Newton-Raphson method to iterate the values of $s$ and $\hat{\phi}^{\prime \prime}(0)$ to satisfy the stress-free boundary conditions $\tilde{\phi}(\pi)=0$ and $\tilde{\phi}^{*}(\pi)=0$.

The convergence of the iteration for the two-parameter shooting method depends critically upon the initial estimate of $s$ and $\bar{\phi}^{* \prime}(0)$. We select $s=(2 n+1) /(n+1)$ as the initial estimate for the eigenvalue $s$. We start our iteration at $n=1$. This gives us the elastic solution for a check for our numerical procedure. Then we use the value of $\tilde{\phi}^{* *}(0)$ of the convergent solution for a higher hardening material for the initial estimate of $\tilde{\phi}^{*}(0)$ for a slightly lower hardening material. Generally speaking, after a few iterations, a convergent solution can be found for each $n$. We normalize our solutions such that the maximum value of the $\theta$-variation $\tilde{S}_{e}$ is set at unity. Similarly, for mode II, solutions can be found by integrating from $\theta=\pi$ to $\theta=0$ since shooting is more stable this way. In this paper, we only present the perturbation solutions for mode I and mode II.

\subsection{Numerical results}

Figure 7 ( $a$ and $b$ ) shows the mode I crack-tip stress solutions perturbed from mode III for $n=3$ and $n=10$, respectively. For comparison, Fig. 8 (a and b) shows the pure mode I crack-tip stress solutions for $n=3$ and $n=10$, respectively. For $n=3$, the angular functions of the perturbed mode I solution in Fig. 7(a) differ from those of the pure mode I solution in Fig. 8 (a). For $n=10$, the two solutions are very different, as shown in Figs 7(b) and 8(b). For example, in Fig. 7(b), the angular function $\tilde{\sigma}_{r r}$ turns up at large $\theta$, the angular functions $\tilde{\sigma}_{r r}$ and $\tilde{\sigma}_{\theta \theta}$ are close to each other at small $\theta$, and the hump of the angular function $\tilde{\sigma}_{r \theta}$ shifts to the right, when compared to those of the pure mode I solution in Fig. 8(b).

In Table 3, the values of $s$ and $t$ are listed for various $n \mathrm{~s}$. We seek the mode I solutions perturbed from mode III. Therefore, the values of $t$ for the out-of-plane shear mode are the HRR values $[(2 n+1) /(n+1)]$. For $n=1$, the numerical solution of $s$ is equal to the HRR value $\frac{3}{2}$. The angular stress functions for $n=1$ are identical to the linear elastic solutions for mode I. As indicated in the table, the eigenvalue $s$ is slightly less than the corresponding HRR value for each $n$ larger than 1 . The values of $s-2$ and $t-2$ are also listed in Table 3 ; these represent the degree of singularity for the in-plane and out-of-plane shear stresses.

As indicated in the table, the mode I crack-tip stresses are slightly more singular than the mode III crack-tip stresses. This characteristic also has been demonstrated in the finite element computations of PAN and SHIH (1990). In addition, the mode III solutions perturbed from mode I also exhibit the same characteristic. Under near 


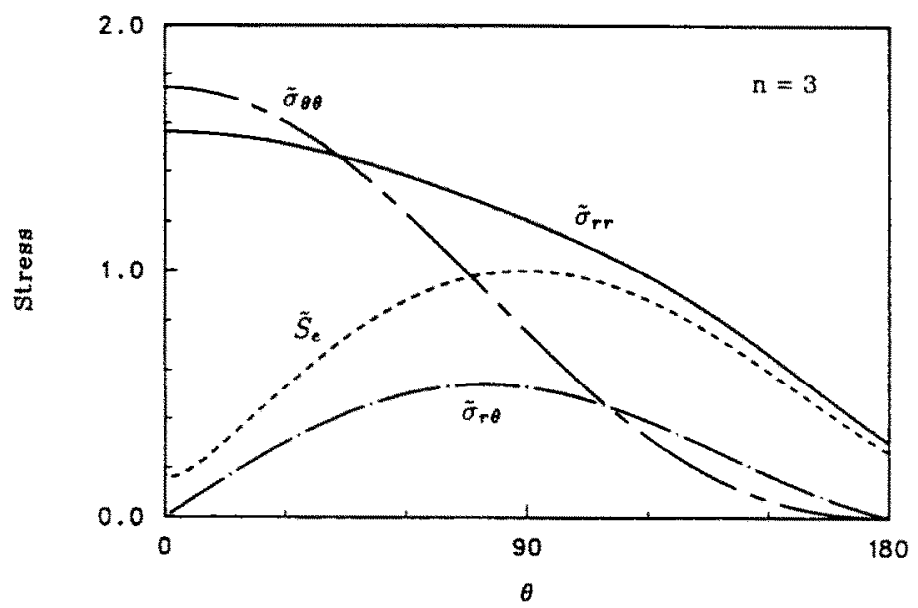

(a)

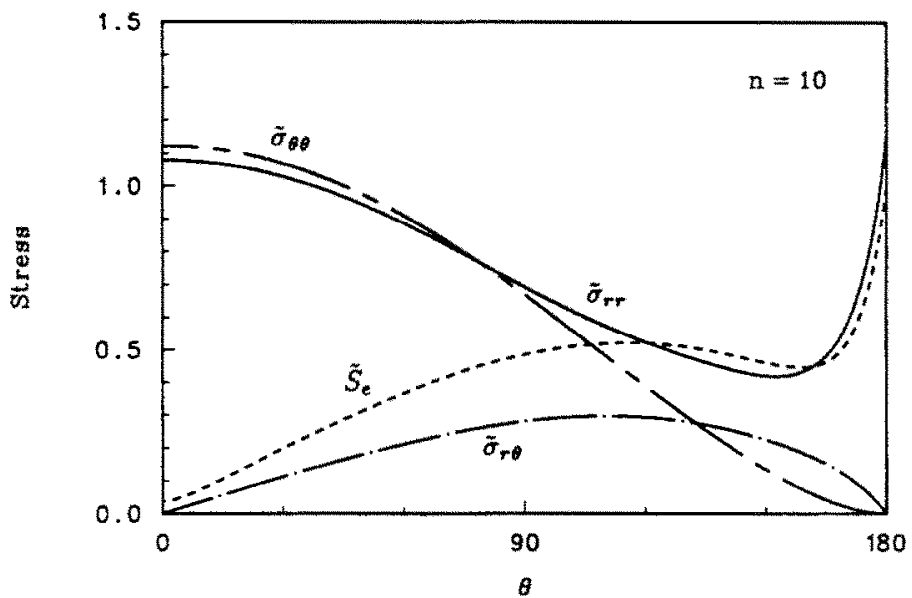

(b)

FrG. 7. The mode I crack-tip stress solutions perturbed from mode III. (a) $n=3$. (b) $n=10$.

mode III combined mode I and III conditions, as $r$ decreases, within the plastic zone the magnitude of the in-plane stresses increases compared to that of the out-of-plane shear stresses due to the stronger singularity of the mode I in-plane stresses. Therefore, the perturbation solutions presented here are only valid in the certain ranges of small $r$ within the plastic zone where the mode III crack-tip fields dominate (or $(K / L)^{2}$ is small).

In Fig. 9 ( $a$ and $b$ ), the normalized mode $I$ in-plane stresses at the Gauss points at about $r / r_{p} \approx 10^{-3}$ are plotted under remote loading of $K_{1} / K_{11}=0.27$ from the finite element computations of PAN and SHIH (1990). The angular functions of the mode I in-plane stresses shown in Fig. 9 (a) for $n=3$ closely resemble those shown in Fig. 


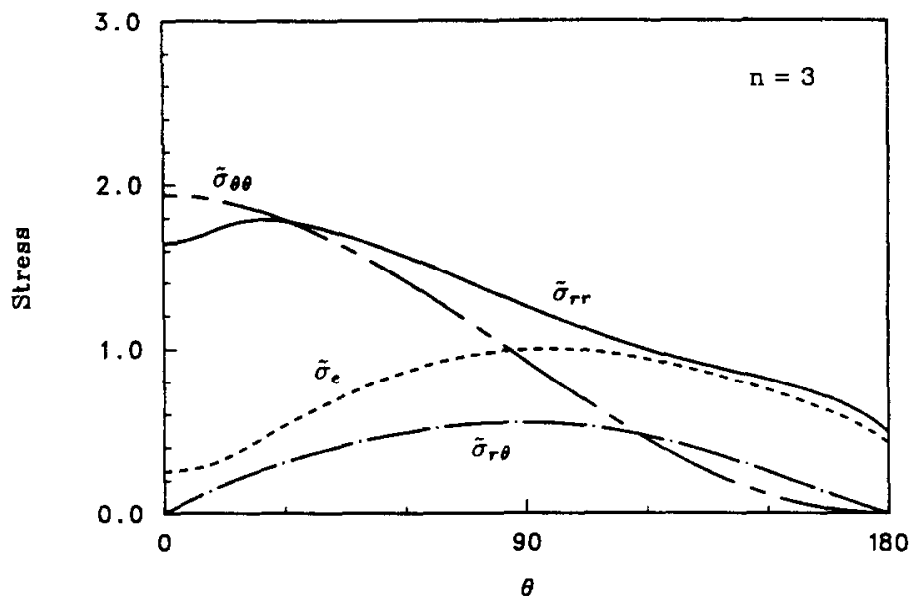

(a)

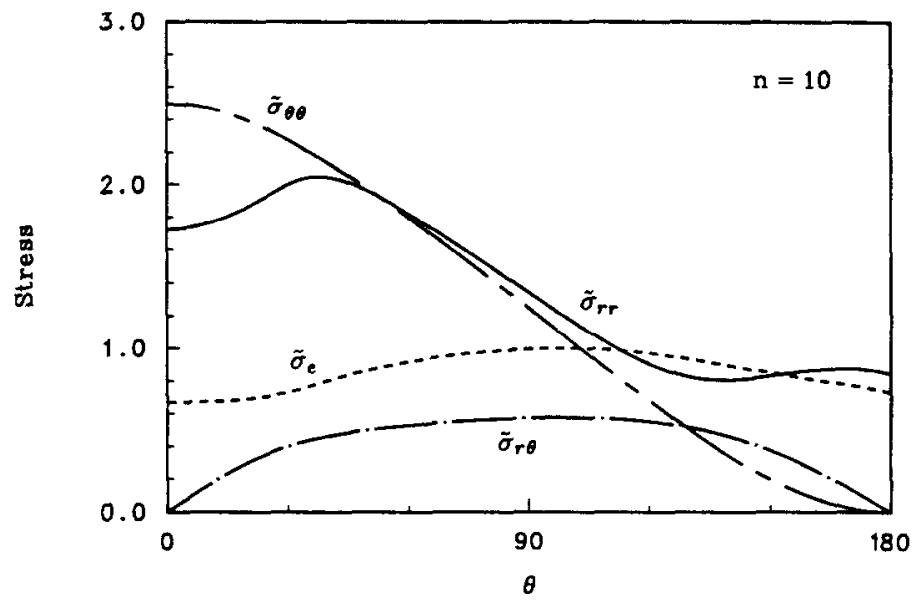

(b)

FIG. 8. The pure mode I crack-tip stress solutions. (a) $n=3$, (b) $n=10$.

TABLE 3. The values of $\mathrm{s}$ for mode I perturbed from mode $I I I[\mathrm{t}=(2 \mathrm{n}+1) /(\mathrm{n}+1)]$

\begin{tabular}{rcccc}
\hline \multicolumn{1}{c}{$n$} & $s$ & $s-2$ & $t$ & $t-2$ \\
\hline 1 & 1.5000 & -0.5000 & 1.5000 & -0.5000 \\
2 & 1.6558 & -0.3442 & 1.6667 & -0.3333 \\
3 & 1.7237 & -0.2763 & 1.7500 & -0.2500 \\
5 & 1.7848 & -0.2152 & 1.8333 & -0.1667 \\
10 & 1.8382 & -0.1618 & 1.9091 & -0.0909 \\
\hline
\end{tabular}




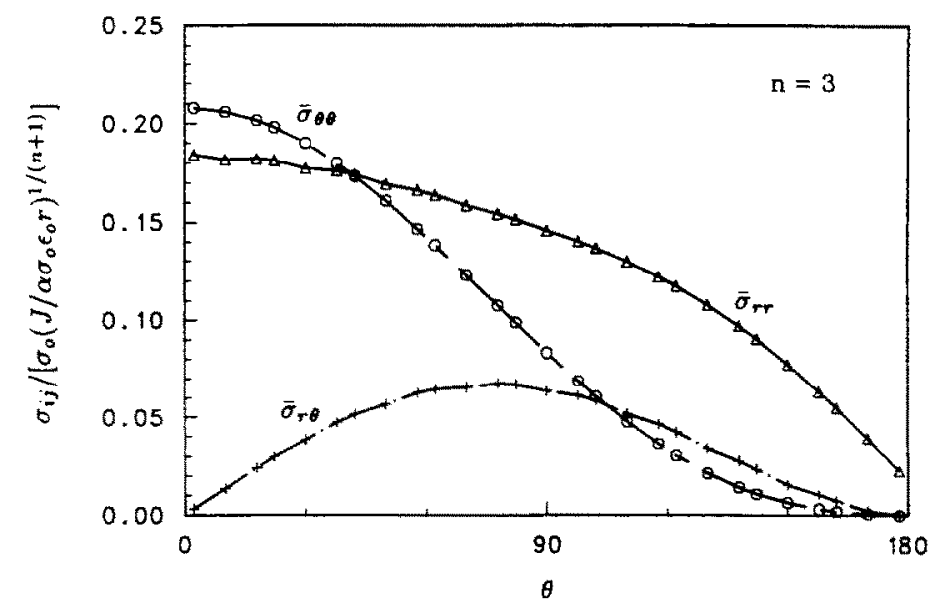

(a)

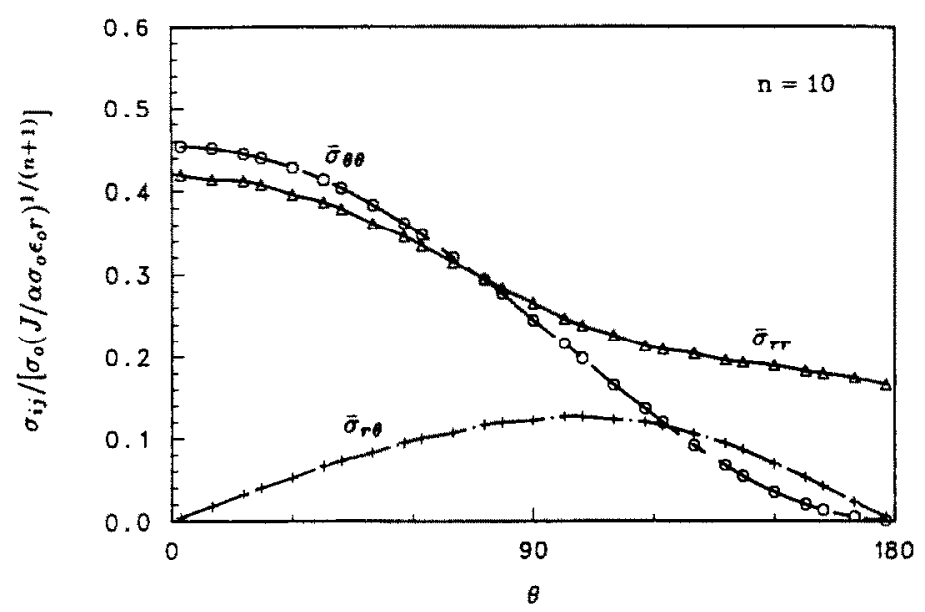

(b)

FiG. 9. The normalized mode I crack-tip stress solutions at $r / r_{p} \approx 10^{-3}$ from finite element computations under remole combined mode I and III conditions of $K_{1} / K_{11}=0.27$. (a) $n=3$. (b) $n=10$.

7(a). The angular functions shown in Fig. 9(b) for $n=10$ also closely resemble those shown in Fig. $7(\mathrm{~b})$, except the upturned $\tilde{\sigma}_{r r}$ at large $\theta$.

The ratio $\sigma_{t \theta} / \sigma_{\theta=}$ at $\theta=0^{\circ}$ is about 0.27 for the remote $K$ field. At a small radial distance to the tip, $r / r_{p} \approx 10^{-3}$, the ratio $\sigma_{\theta \theta} / \sigma_{\theta=}$ at $\theta=0^{n}$ is about 0.45 for $n=3$ and about 0.84 for $n=10$. The results indicate that due to material plasticity, within the plastic zone the ratio of the magnitude of the mode I in-plane stresses to the magnitude of the mode III out-of-plane shear stresses increases as $r$ decreases. The stronger singularity of mode I in-plane stresses compared to that of the mode III out-of-plane shear stresses agrees with the results of our perturbation analysis. This effect is more 


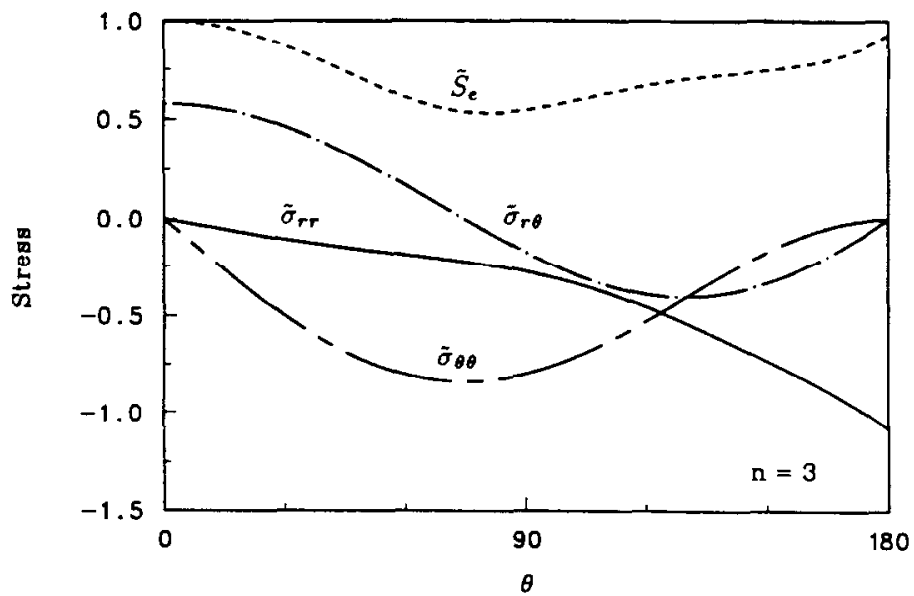

(a)

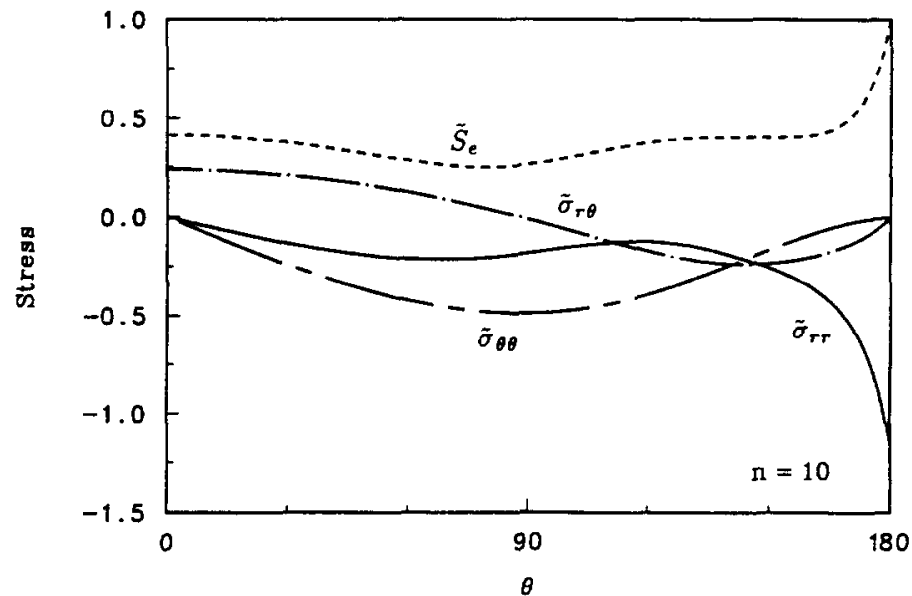

(b)

FIG. 10. The mode II crack-tip stress solutions perturbed from mode III. (a) $n=3$, (b) $n=10$.

pronounced for low-hardening materials (with large $n$ ). Here, we can conclude that for low-hardening materials (for example, $n=10$ ), when there is only a small amount of remote mode I loading, the mode I contribution becomes significant well within the plastic zone due to material plasticity. The estimated values of the singularity difference $t-s$ from the finite element computations for the cases where the contribution of the mode I in-plane stresses to the effective stress is smaller than that of the mode III out-of-plane shear stresses are in good agreement with the values listed in Table 3.

Figure 10 ( $a$ and $b$ ) shows the mode II crack-tip stress solutions perturbed from mode III for $n=3$ and $n=10$, respectively. Figure 11 ( $\mathrm{a}$ and $\mathrm{b}$ ) shows the pure mode 


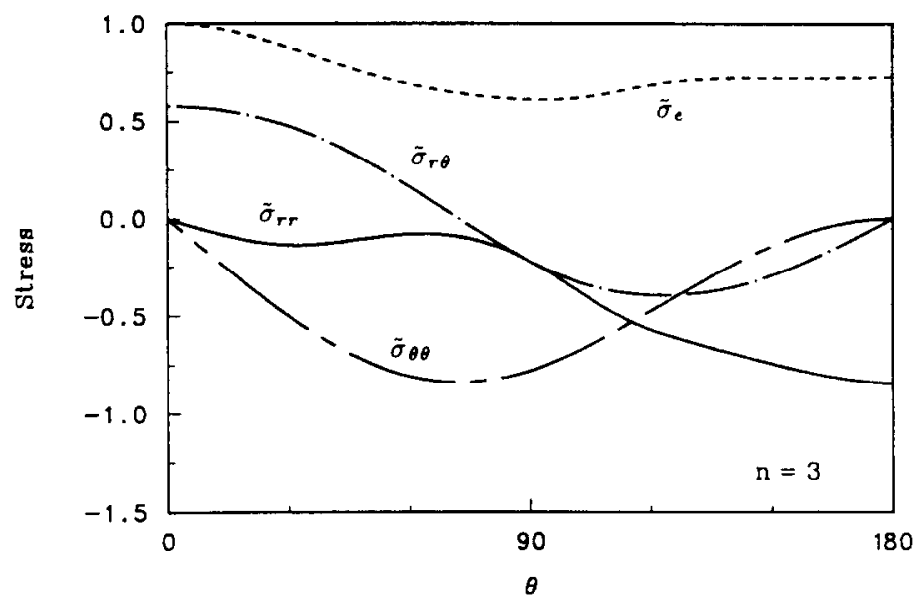

(a)

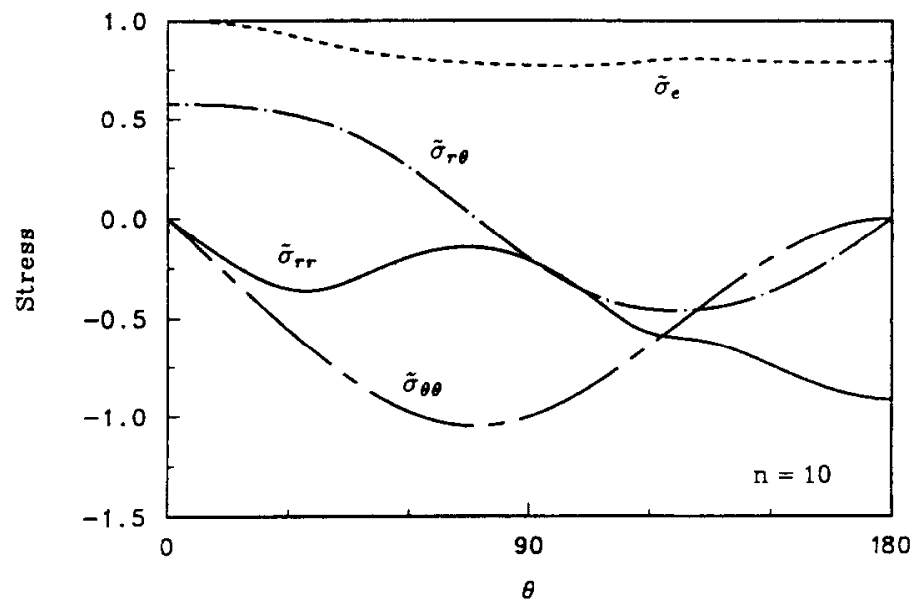

(b)

FiG. 11. The pure mode II crack-tip stress solutions. (a) $n=3$, (b) $n=10$.

II crack-tip stress solutions for $n=3$ and $n=10$, respectively. In Figs 10 (a) and 11 (a) for $n=3$, the main difference is the angular variation of $\tilde{\sigma}_{r r}$ for the perturbed solutions. This feature is more pronounced for $n=10$ in Figs $10(\mathrm{~b})$ and 11 (b).

In Table 4, the values of $s$ and $t$ are listed for various $n$ s. Note that the values of $t$ are the HRR values $[(2 n+1) /(n+1)]$, since we seek the solutions perturbed from mode III. As indicated in the table, the value of $s$ is slightly larger than that of $t$ for each $n$ larger than 1 . This implies that the singularity of the mode II crack-tip stresses is weaker than that of the mode III crack-tip stresses. Note that this characteristic is also shown in the mode III solutions perturbed from mode II. Note that for $n=10$ 
TABLE 4 . The values of $\mathrm{s}$ for mode II perturbed from

mode $I I I[\mathrm{t}=(2 \mathrm{n}+1) /(\mathrm{n}+1)]$

\begin{tabular}{rcccc}
\hline$n$ & $s$ & $s-2$ & $t$ & $t-2$ \\
\hline 1 & 1.5000 & -0.5000 & 1.5000 & -0.5000 \\
2 & 1.6751 & -0.3246 & 1.6667 & -0.3333 \\
3 & 1.7701 & -0.2299 & 1.7500 & -0.2500 \\
5 & 1.8752 & -0.1248 & 1.8333 & -0.1667 \\
10 & 1.9973 & -0.0027 & 1.9091 & -0.0909 \\
\hline
\end{tabular}

the value of $s$ becomes very close to 2 . In fact, the numerical solutions give a value of $s$ larger than 2 for $n$ larger than 10 . When $s$ is larger than 2 , the singularity of the mode II in-plane stresses disappears. This interesting feature indicates that the singularity of the mode II in-plane stresses is very weak and may disappear for lowhardening materials under near mode III combined mode II and III conditions.

Figure 12 ( $\mathrm{a}$ and $\mathrm{b}$ ) shows the normalized mode II in-plane stresses at the Gauss points at about $r / r_{p} \approx 10^{-3}$ under remote loading of $K_{\mathrm{II}} / K_{\mathrm{II}}=0.27$ from the finite element computations of PAN and SHIH (1989). The angular functions of the mode II in-plane stresses shown in Fig. 12 ( $a$ and $b$ ) closely resemble those of the perturbation solutions shown in Fig. 10 ( $a$ and $b$ ).

The ratio $\sigma_{r \theta} / \sigma_{\theta z}$ at $\theta=0^{\circ}$ is about 0.27 for the remote $K$ field. At a small radial distance to the tip, $r / r_{p} \approx 10^{-3}$, the ratio $\sigma_{r \theta} / \sigma_{\theta_{z}}$ at $\theta=0^{\circ}$ is about 0.30 for $n=3$ and about 0.24 for $n=10$. Note that the ratio $\sigma_{r \theta} / \sigma_{\theta z}$ at $\theta=0^{\circ}$ decreases to about 0.22 for $n=3$ and about 0.14 for $n=10$ at about $r / r_{p} \approx 10^{-11}$. The results indicate that well within the plastic zone $\left(r / r_{p}<10^{-3}\right)$ the singularity of the mode II in-plane stresses is weaker than that of the mode III out-of-plane shear stresses. This characteristic agrees with the results of the perturbation analysis. The reason that the ratio $\sigma_{r \theta} / \sigma_{\theta z}$ for $n=3$ at $\theta=0^{\circ}$ increases a bit in the range of $r / r_{p}=1$ to $10^{-3}$ as $r$ decreases is perhaps due to the transition of the elastic state to the plastic state as mentioned earlier. Generally speaking, these finite element computations show that due to material plasticity, well within the plastic zone the ratio of the magnitude of the mode II in-plane stresses to the magnitude of the mode III out-of-plane shear stresses decreases as $r$ decreases. The stronger singularity of the mode III out-of-plane stresses comparcd to that of the mode II in-plane shear stresses agrees well with the results of our perturbation analysis. The estimated values of the singularity difference $t-s$ from the finite element computations for the cases where the contribution of the mode II in-plane stresses to the effective stress is smaller than that of the mode III out-of-plane shear stresses are in good agreement with the values listed in Table 4.

\section{Discussion and Conclusions}

In this paper, we present the solutions for the asymptotic crack-tip stresses based on the perturbation of the governing equations derived from the stress functions $\phi$ and $\psi$ in (3.3) and (3.4). These perturbation solutions are only approximately valid 


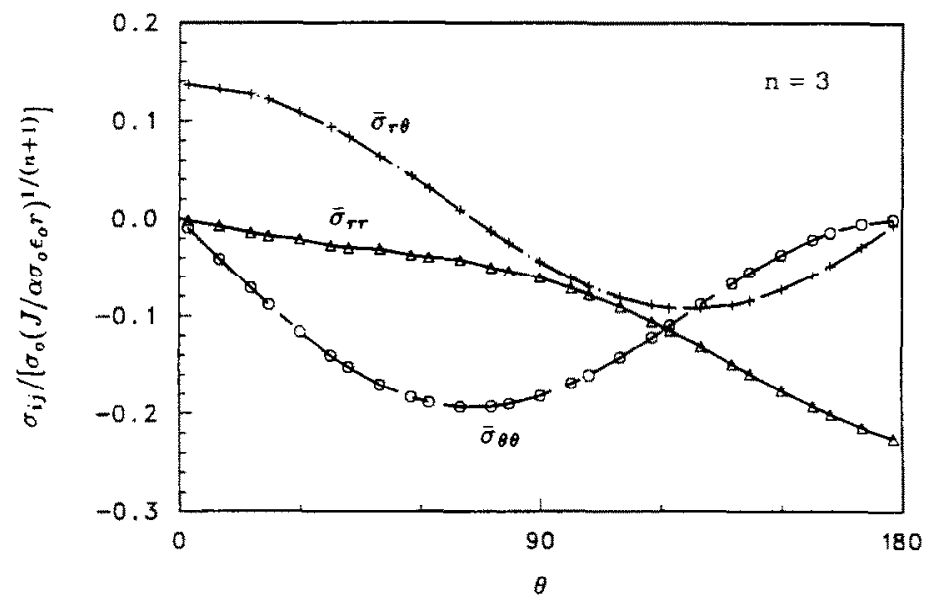

(a)

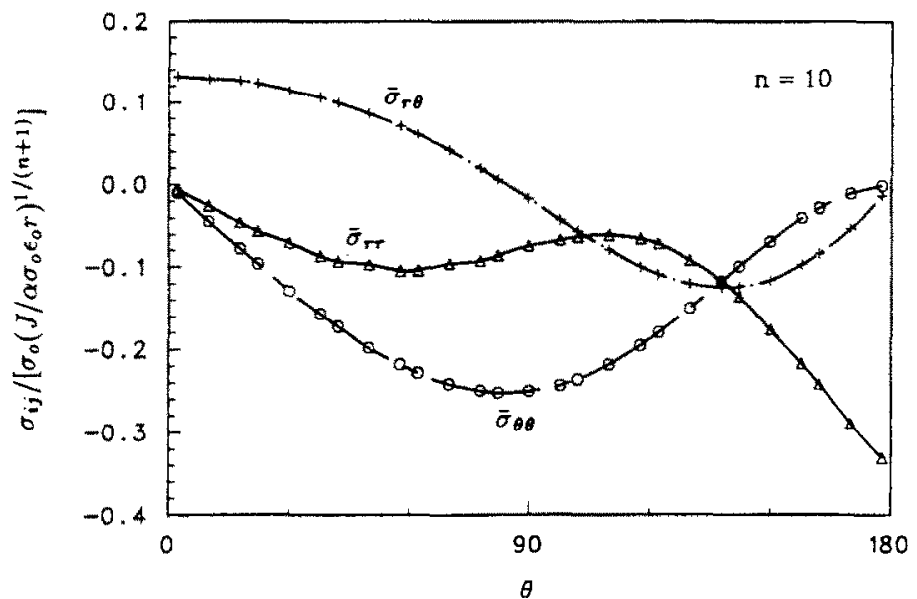

(b)

FIG. 12. The normalized mode 11 crack-tip stress solutions at $r / r_{p} \approx 10^{-3}$ from finite element computations under remote combined mode II and III conditions of $K_{\mathrm{y}} / K_{\mathrm{m}}=0.27$. (a) $n=3$, (b) $n=10$.

in the ranges of small $r$ within the plastic zone where either the in-plane mode or the out-of-plane shear mode is smaller than the other. These solutions provide a partial picture of the near-tip fields under combined in-plane and out-of-plane shear conditions. The complete picture of the near-tip fields must be obtained by full-field analyses. But these perturbation solutions do provide a correct qualitative understanding of the combined-mode near-tip fields.

Specifically, according to the perturbation solutions, under combined mode I and III conditions, the singularity of the mode I crack-tip stresses is stronger than that of 
the mode III crack-tip stresses when the contribution of either of the crack-tip stresses to the effective stress is smaller than that of the other. The results of the finite element computations (PAN and SHIH, 1990) confirm that under the complete range of combined mode I and III conditions, within the plastic zone the singularity of the mode I crack-tip stresses is stronger than that of the mode III crack-tip stresses. Furthermore, the angular functions of the mode I (or mode III) crack-tip stresses at a small radial distance (to the tip), where the contribution of the mode I (or mode III) stresses to the effective stress is small compared to that of the mode III (or mode I) stresses, agree well with the corresponding perturbation solutions.

Similarly, according to the perturbation solutions, under combined mode II and III conditions, the singularity of the mode III crack-tip stresses is stronger than that of the mode II crack-tip stresses when the contribution of either of the crack-tip stresses to the effective stress is smaller than that of the other. The results of the finite element computations (PAN and SHIH, 1989) confirm that under the complete range of combined mode II and III conditions, well within the plastic zone the singularity of the mode III crack-tip stresses is stronger than that of the mode II crack-tip stresses. Furthermore, the angular functions of the mode II (or mode III) crack-tip stresses at a small radial distance (to the tip), where the contribution of the mode II (or mode III) stresses to the effective stress is small compared to that of the mode III (or mode II) stresses, agree well with the corresponding perturbation solutions.

The results of the finite element computations of PAN and SHIH $(1989,1990)$ also gave the estimated values of the singularity difference of the in-plane or out-of-plane shear stresses from the HRR singularity under combined in-plane and out-of-plane shear conditions. These values for the cases where either the contribution of the inplane stresses or the out-of-plane shear stresses to the effective stress is smaller than that of the other agree with the results listed in Tables 1-4. The angular functions of the stresses of the finite element computations closely resemble the perturbation results when either the in-plane mode or the out-of-plane shear mode is smaller than the other. For example, the angular functions of the out-of-plane shear stresses at a small fixed radial distance to the tip within the plastic zone approach the perturbation results when $K_{\mathrm{III}} / K_{1}$ decreases. The angular functions also approach the perturbation results as $r / r_{p}$ decreases under a fixed $K_{\mathrm{III}} / K_{\mathrm{l}}$. Under a fixed $K_{\mathrm{III}} / K_{\mathrm{l}}$, if the angular functions resemble the perturbation results at a small $r / r_{p}$ where the out-of-plane shear mode is smaller than the in-planc mode, then the angular functions are virtually insensitive to the further decrease of $r / r_{p}$.

We have presented the crack-tip stresses at a small radial distance of about $r / r_{p} \approx 10^{-3}$ to show the effect of plasticity on the crack-tip fields using finite element methods. Our results from the finite element computations at smaller radial distances $\left(r / r_{p}<10^{-3}\right)$ for larger ratios of the remote in-plane $K$ field to the remote out-ofplane shear $K$ field (or the remote out-of-plane shear $K$ field to the remote in-plane $K$ field) give the same qualitative results as those presented here. However, the smallstrain approach is perhaps valid at the radial distance of $r / r_{p} \approx 10^{-3}$ at the most for common dense metals and alloys. For these materials, the crack-tip stresses at $r / r_{p} \approx 10^{-3}$ provide important information for fracture analyses since they represent the stresses at a length scale of the crack-tip fracture process zone in which finite deformation effects must be considered. We emphasize here that our intent is to 
demonstrate the mathematical structure of the crack-tip fields within the context of the small-strain continuum approach. The results of these finite element computations also have indicated that there is no other unexpected pathological stress and strain behavior at $r / r_{p}<10^{-3}$.

Under combined in-plane and out-of-plane shear loading conditions, when either one of the in-plane modes or the out-of-plane shear mode is more singular than the other, mathematically speaking, as $r / r_{p}$ approaches 0 , the more singular mode (either the in-plane mode or the out-of-plane shear mode) will dominate. Then the HRRtype of singular behavior for the pure in-plane mode or the pure out-of-plane shear mode should be recovered. However, as shown in both the asymptotic analysis and the finite element computations of PAN and SHIH $(1989,1990)$, the singularity of the in-plane modes is only slightly different from that of the out-of-plane shear mode. Furthermore, as shown in the finite element computations of PAN and SHIH (1989. 1990 ), at a small radial distance of about $r / r_{p} \approx 10^{-3}$, the magnitude of the crack-tip stresses of the less singular mode is comparable to that of the more singular mode when the remote elastic in-plane and out-of-plane shear $K$ fields have the same order of magnitude. Therefore, under combined in-plane and out-of-plane shear loading conditions, the pure in-plane mode and the pure out-of-plane shear mode HRR fields only exist as the limit cases as $r / r_{p} \rightarrow 0$.

Within the plastic zone at a small radial distance (to the tip) of practical interest, we should take into account the different singular behaviors of the in-plane and out-of-plane shear stresses under combined in-plane and out-of-plane shear loading conditions. For engineering applications, we may use the values of $t-s$ listed in Tables 1-4 in combination with the HRR crack-tip field to estimate the crack-tip stresses at a radial distance (to the tip) of microstructural relevance for fracture analysis.

\section{ACKNOWLEDGEMENT}

The support of this work by the National Science Foundation under Grant No. MSM8613544 at The University of Michigan is acknowledged. Helpful discussions with Professor C. F. Shih of Brown University and Professor J. R. Rice of Harvard University on this subject are greatly appreciated.

\section{REFERENCES}

Dung, P. and Pan, J.

DONG, P. and PAN, J.

HAYASHI, K.

HILL, R.

HUTCHINSON, J. W. HUTCHINSON, J. W. 1989a Plane strain mixed mode near-lip felds in elastic perfectly plastic solids under small-scale yielding conditions, submitted for publication in Int. J. Fracture.

1989b Asymptotic crack-tip fields for perfectly plastic solids under plane-stress and mixed-mode loading conditions, submitted for publication in J. appl. Mech.

1979 J. Mech. Phys. Solids 27, 163.

1948 Proc. R. Soc. (Lond.) A193, 281.

1968 a J. Mech. Phys. Solids 16, 13.

1968 b J. Mech. Phys. Solids 16, 337. 
HUTCHINSON, J. W.

LI, F. Z. and PAN, J.

LI, F. Z. and PAN, J.

PAN, J.

PAN, J.

PAN, J. and SHIH, C. F.

PAN, J. and SHIH, C. F.

PAN, J. and SHIH, C. F.

PAN, J. and SHIH, C. F.

RICE, J. R.

RICE, J. R.

RICE, J. R.

RICE, J. R.

Rice, J. R. and Nikolic R.

RICE, J. R. and Rosengren, G. F. 1968

SHIH, C. F.

SHIH, C. F.

1986

1988

1986

1988

1989

1990

1967

1987

1985
1983 J. appl. Mech. 50, 1042.

1989a Plane-strain crack-tip fields for pressure-sensitive dilatant materials, to appear in J. appl. Mech.

$1989 \mathrm{~b}$ Plane-stress crack-tip fields for pressure-sensitive dilatant materials, to appear in Engng Fracture Mech.

$1968 \mathrm{a}$

$1968 \mathrm{~b}$

J. Mech. Phys. Solids 34, 617.

Int. J. Fracture 38, 103.

Mech. Mater. 5, 299.

Int. J. Fracture 37, 171.

Elastic-plastic analysis of combined mode II and III crack-tip fields under small-scale yielding conditions, to appear in J. appl. Mech.

J. Mech. Phys. Solids 38, 161.

J. appl. Mech. 34, 287.

J. appl. Mech. 35, 379.

In Fracture, Vol. 2 (edited by H. Liebowitz), pp. 191-311. Academic Press, New York, NY.

Tensile crack tip fields in elastic-ideally plastic crystals, Mech-106, Division of Applied Sciences, Harvard University, Cambridge, MA.

J. Mech. Phys. Solids 33, 595.

J. Mech. Phys. Solids 16, 1.

Elastic-plastic analysis of combined mode crack problems, Ph.D. Thesis, Harvard University, Cambridge, MA.

1974 Fracture Analysis, ASTM STP 560, 187. 